\title{
C-Reactive Protein/Albumin Ratio and Prognostic Nutritional Index Are Strong Prognostic Indicators of Survival in Resected Pancreatic Ductal Adenocarcinoma
}

Masahide Ikeguchi, ${ }^{*}$ Takehiko Hanaki, Kanenori Endo, Kazunori Suzuki, Seiichi Nakamura, Takashi Sawata, and Tetsu Shimizu

\begin{abstract}
Purpose: We evaluated the clinical importance, such as the occurrence of postoperative pancreatic fistula (POPF) or prognosis, of preoperative serum markers of chronic inflammation, nutrition, and immunity, as well as that of serum tumor markers after curative resection of pancreatic ductal adenocarcinomas (PDACs).

Methods: Between 2006 and 2015, 43 patients with PDACs underwent curative resection at Tottori Prefectural Central Hospital. We analyzed which preoperative indicators (i.e., C-reactive protein/albumin ratio [CAR], neutrophil/lymphocyte ratio [NLR], prognostic nutritional index [PNI], carcinoembryonic antigen [CEA], and carbohydrate antigen 19-9 [CA 19-9]) were the most relevant risk factors for occurrence of POPF and poor patient survival. Results: POPF was detected in 8/43 (18.6\%) patients. One patient died of pancreatic fistula at 2 months postoperatively. Among nine candidate factors (operative procedure, operation time, tumor stage, preoperative serum amylase, preoperative CAR, NLR, PNI, CEA, and CA 19-9), we did not identify any significant risk factor for the occurrence of POPF. The 5-year overall survival (OS) rate of the 43 patients was $22.4 \%$, and the overall median survival time was 21 months. The multivariate OS analysis demonstrated that high CAR and low PNI were strong preoperative markers of poor prognosis independently of tumor stage.
\end{abstract}

Conclusions: Preoperative CAR and PNI are useful prognostic markers for patients with operable PDACs.

Keywords: C-reactive protein/albumin ratio; overall survival; pancreatic ductal adenocarcinoma; postoperative pancreatic fistula; prognostic nutritional index

\section{Introduction}

The prognosis of patients with pancreatic ductal adenocarcinomas (PDACs) is extremely poor. Even though curative resection is performed, many patients die of cancer recurrence within a few months. ${ }^{1-3}$ Yamamoto et al. ${ }^{4}$ reported that the median survival of 195 patients who underwent pancreatic resection was 27.1 months, and the 5-year actuarial survival rate was $34.5 \%$. In addition, Sato et al. $^{5}$ reported that in patients with resected PDACs in Japan, the estimated 5-year disease- specific survival rate was $23.1 \%$, and the disease-free survival (DFS) rate was $16.8 \%$. Moreover, it has been reported that after resection of the pancreas, postoperative complications occur very frequently. Anastomotic leakages between the pancreatic duct and the jejunum have been observed in $14-17 \%$ of patients after pancreatoduodenectomy (PD) ${ }^{6-8}$ In addition, pancreatic fistula is frequently detected (16-26\%) after distal pancreatectomy (DP). ${ }^{9-11}$ Such anastomotic leakages or pancreatic fistula are often fatal.

Department of Surgery, Tottori Prefectural Central Hospital, Tottori, Japan.

*Address correspondence to: Masahide Ikeguchi, MD, Department of Surgery, Tottori Prefectural Central Hospital, 730 Ezu, Tottori 680-0901, Japan, E-mail: ikeguchim@ pref.tottori.lg.jp

(c) Masahide Ikeguchi et al. 2017; Published by Mary Ann Liebert, Inc. This is an Open Access article distributed under the terms of the Creative Commons Attribution License, which permits unrestricted use, distribution, and reproduction in any medium, provided the original work is properly cited. 
Recently, several studies found a strong correlation between poor survival and preoperative chronic inflammation, poor nutrition, and low immunity among patients with various cancers. ${ }^{12-14}$ Patient preoperative C-reactive protein/albumin ratio (CAR) has been used in combination with other parameters not only to diagnose chronic inflammation but also to assess the nutritional status of patients with cancer. Neutrophil/lymphocyte ratio (NLR) and prognostic nutritional index (PNI) are markers of chronic systemic inflammation and the patients' immune status and nutritional condition. ${ }^{15,16}$ Also, serum carcinoembryonic antigen (CEA) and carbohydrate antigen 19-9 (CA 19-9) levels are known to be good progression indicators among patients with PDACs, including followup after resection of tumors. ${ }^{1,17}$

In the present study, we evaluated the prognostic and clinical importance of serum markers of chronic inflammation, nutrition, and immunity combined with tumor markers of patients with PDACs.

\section{Patients and Methods}

Patients

Between 2006 and 2015, we enrolled 43 patients who were diagnosed with operable PDAC and underwent curative resection (no residual tumors macroscopically) at Tottori Prefectural Central Hospital. The average age of the 30 male and 13 female patients was 71.6 (range: 3788) years. Clinical and pathological staging of PDACs was performed using the American Joint Committee on Cancer 6th edition tumor-node-metastasis (TNM) staging system for pancreatic cancer. ${ }^{18}$ All patients were followed up at Tottori Prefectural Central Hospital until the end of 2016. The mean follow-up period was 25.2 (range: 1-80) months. No patients received chemotherapy and radiation therapy before the operation. Informed consent for medical treatment and use of clinical data from medical records were obtained from all patients.

\section{Surgical procedure}

All patients underwent open laparotomy. According to the tumor location, we performed DP with splenectomy in 14 patients, subtotal stomach-preserving pancreaticoduodenectomy (SSPPD) in 24 patients, SSPPD combined with portal vein partial resection in 4 patients, and total pancreatectomy with splenectomy in 1 patient. After DP, we closed the proximal pancreas stump by suturing with 4-0 prolene in 12 patients and by linear stapler in 2 patients. Reconstruction after SSPPD or SSPPD with partial resection of the portal vein was performed by Child's method (e.g., Braun's anastomosis after pancreaticojeju- nostomy, choledochojejunostomy, and gastrojejunostomy). ${ }^{19}$ An internal short stent was placed across a pancreaticojejunostomy, but no stent was placed following choledochojejunostomy.

\section{Postoperative complications}

Postoperative complications were analyzed by patient clinical record data. The severity of postoperative complications was graded according to the Clavien-Dindo classification. ${ }^{20}$ In addition, a Clavien-Dindo score of III or higher was considered a major complication.

\section{Study design}

This study was a retrospective study. We recorded clinical parameters, including operation time, postoperative hospital stay, and occurrence of postoperative complications. Blood samples were taken from each patient routinely at the time of admission to our hospital. C-reactive protein, serum albumin, serum amylase, total lymphocyte count, and tumor markers, (including CEA and CA 19-9), were recorded. In addition, CAR, NLR, and PNI were calculated. The PNI was calculated using the following formula: $10 \times$ serum albumin concentration $(\mathrm{g} / \mathrm{dL})+0.005 \times$ lymphocyte counts $\left(\right.$ no. $\left./ \mathrm{mm}^{3}\right)$ in peripheral blood. ${ }^{15}$ The reference ranges of CAR, NLR, and PNI were determined by investigating the preoperative CAR, NLR, and PNI in 147 patients who underwent radical inguinal hernia operations from 2012 to 2015 at our hospital. None of these patients had either chronic or acute inflammation. The average age of the 147 control patients was 69 years (range: 27-93 years). Ninety-two were male, and 55 were female. The mean CAR, NLR, and PNI among of the 147 control patients were 0.037 (range: 0.002-0.464), 2.5 (range: 0.9-19.4), and 49.9 (range: $35.2-61.7$ ), respectively. To determine the cutoff values corresponding to the CAR, NLR, and PNI, we plotted receiver operating characteristic (ROC) curves for the 147 control patients and 43 patients with PDACs. The Youden index (sensitivity + specificity -1 ) was used, and the highest Youden index values were considered the cutoff levels. ${ }^{21}$ According to a previous report, the normal range of serum amylase, CEA, and CA 19-9 levels were $37-125 \mathrm{U} / \mathrm{L}, 0-5 \mathrm{ng} / \mathrm{mL}$, and $0-37 \mathrm{U} / \mathrm{mL}$, respectively. ${ }^{22}$ This study was approved by the Ethical Review Board of Tottori Prefectural Central Hospital (approval number: 2016-49).

\section{Statistical analysis}

Differences between two normally distributed parameters were compared using the $\chi^{2}$ and Fisher's exact 
probability tests. The Mann-Whitney $U$ test was used to compare differences between two parameters with nonnormal distribution. Correlations between the parameters and occurrence of postoperative pancreatic fistula (POPF) were estimated using logistic-regression analysis. Long-term OS was calculated using the Kaplan-Meier method and the prognostic difference between the two groups was compared using the logrank test. All data were analyzed by StatView software (Abacus Concepts, Inc., Berkeley, CA). $p<0.05$ was regarded as indicating statistical significance.

\section{Results}

The clinical characteristics, operative procedure, operation time, postoperative complications, and postoperative hospital stay of all 43 patients with PDACs are presented in Table 1. Postoperative complications were detected in $34.9 \%$ of cases. POPF secondary to pancreatic juice leakage at the anastomotic site or at the pancreas stump was frequently detected $(8 / 43,18.6 \%)$. The mean length of postoperative hospital stay of the 15 patients with postoperative complications (61.4 days) was significantly longer than that of the 28 patients without postoperative complications (22.2 days, $p<0.001)$. One patient died from postoperative peritonitis secondary to POPF 2 months postoperatively. Thus, the operative mortality and morbidity in our series were $2.3 \%$ and $34.9 \%$, respectively.

The mean and median (range) of preoperative serum amylase, CAR, NLR, PNI, CEA, and CA 19-9 of all 43 patients with PDACs were as follows: serum amylase

Table 1. Sample Characterizations $(n=43)$

\begin{tabular}{lc}
\hline Parameters & \\
\hline Age (years, mean, range) & $71.6(37-88)$ \\
Gender (male, \%) & $30(69.8)$ \\
Operative procedures (\%) & \\
DP & $14(32.6)$ \\
SSPPD & $24(55.8)$ \\
SSPPD combined with portal vein partial resection & $4(9.3)$ \\
Total pancreatectomy with splenectomy & $1(2.3)$ \\
Operation time: min (mean, range) & $324.7(150-568)$ \\
Pathological tumor stages (\%) & \\
IA & $3(7)$ \\
IB & $2(4.7)$ \\
IIA & $7(16.3)$ \\
IIB & $25(58.1)$ \\
III & $6(14)$ \\
Postoperative complications & \\
None & 28 \\
Yes (pancreatic fistula, passage disorder, others) & $15(8 / 3 / 4)$ \\
Postoperative hospital stay: day (mean, range) & $35.9(10-240)$ \\
\hline
\end{tabular}

DP, distal pancreatectomy; SSPPD, subtotal stomach-preserving pancreaticoduodenectomy.
(111.7, 76, [42-399] U/L), CAR (0.11, 0.05, [0.010.57]), NLR (2.3, 2.2, [0.9-5.2]), PNI (47.7, 48.8, [29.4-63.4]), CEA (7.7, 4.5, [1.1-48.5] ng/mL), and CA 19-9 (369.7, 165, [1-2640] U/mL). According to the cutoff levels of the six parameters, abnormal levels of serum amylase, CAR, NLR, PNI, CEA, and CA 19-9 were detected in 11 (25.6\%), 23 (53.5\%), 18 (41.9\%), 15 (34.9\%), $19(44.2 \%)$, and 31 (72.1\%) of the 43 patients.

We analyzed factors that were thought to influence the occurrence of POPF. The nine candidate factors were operative procedure, operation time, tumor stage, preoperative serum amylase, preoperative CAR, NLR, PNI, CEA, and CA 19-9. We divided the patients into two subgroups (long and short) according to the median values of operation time, but we could not find any significant risk factor for the occurrence of POPF (data not shown).

The 5-year overall survival (OS) rate of the $43 \mathrm{pa}$ tients was $22.4 \%$, and the overall median survival time (MST) was 21 months. In addition, the 5-year DFS rate of the 43 patients was $9.1 \%$, and the diseasefree MST was 9 months. The correlation between the 10 candidate factors (operative procedure, operation time, tumor stage, occurrence of postoperative complications, preoperative serum amylase, preoperative CAR, NLR, PNI, CEA, and CA 19-9) and the overall MST and disease-free MST of 43 patients are shown in Table 2. We found that low preoperative PNI was important risk factor of poor survival in patients with PDACs and, we found that there was even no statistical significance, high preoperative CAR was also risk factor of poor survival. Figure 1 shows the OS curves of 28 patients with high PNI (solid line) and 15 patients with low PNI (dotted line). The 5-year OS of patients with low PNI (4\%) was significantly poorer than that of patients with high PNI $(35 \%, p=0.001)$. The multivariate analysis of OS showed that high CAR and low PNI were strong prognostic preoperative markers independently of tumor stage (Table 3 ). In addition, in multivariate analysis of DFS, we found that high CAR was a significant poor survival and recurrence preoperative marker (CAR: $p=0.018$, odds ratio $=2.948,95 \%$ confidence interval $=1.203-7.222$ ), but low PNI was not (PNI: $p=0.124$, odds ratio $=2.247,95 \%$ confidence interval $=0.802-6.289$ ).

\section{Discussion}

The incidences of severe POPF (Clavien-Dindo score of III or higher) were reported in $14-23 \%$ of patients after $\mathrm{PD}^{23}$ and in $10-30 \%$ of patients after $\mathrm{DP}^{24}$ 
Table 2. Univariate Survival Analyses of 43 Patients

\begin{tabular}{|c|c|c|c|c|c|}
\hline & $N$ & Overall MST (months) & $p$ & Disease-free MST (months) & $p$ \\
\hline \multicolumn{6}{|l|}{ Operative procedures } \\
\hline DP or total pancreatectomy & 15 & 29 & \multirow[t]{2}{*}{0.266} & 12 & \multirow[t]{2}{*}{0.614} \\
\hline SSPPD with or without portal vein resection & 28 & 15 & & 8 & \\
\hline \multicolumn{6}{|l|}{ Operation time } \\
\hline Long >297 min & 21 & 15 & \multirow[t]{2}{*}{0.083} & 7 & \multirow[t]{2}{*}{0.115} \\
\hline Short $\leq 297 \mathrm{~min}$ & 22 & 28 & & 13 & \\
\hline \multicolumn{6}{|l|}{ Tumor stages } \\
\hline Stage I and IIA & 12 & 61 & \multirow[t]{2}{*}{0.247} & 33 & \multirow[t]{2}{*}{0.132} \\
\hline Stage IIB and III & 31 & 18 & & 8 & \\
\hline \multicolumn{6}{|l|}{ Postoperative complication } \\
\hline Yes & 15 & 23 & \multirow[t]{2}{*}{0.658} & 9 & \multirow[t]{2}{*}{0.67} \\
\hline No & 28 & 19 & & 8 & \\
\hline \multicolumn{6}{|l|}{ Preoperative amylase level } \\
\hline High $>125 \mathrm{U} / \mathrm{L}$ & 11 & 15 & \multirow[t]{2}{*}{0.481} & 10 & \multirow[t]{2}{*}{0.994} \\
\hline Low $\leq 125 \mathrm{U} / \mathrm{L}$ & 32 & 24 & & 8 & \\
\hline \multicolumn{6}{|l|}{ Preoperative CAR } \\
\hline High $>0.04$ & 23 & 19 & \multirow[t]{2}{*}{0.06} & 6 & \multirow[t]{2}{*}{0.085} \\
\hline Low $\leq 0.04$ & 20 & 29 & & 12 & \\
\hline \multicolumn{6}{|l|}{ Preoperative NLR } \\
\hline High $>2.5$ & 18 & 19 & \multirow[t]{2}{*}{0.248} & 8 & \multirow[t]{2}{*}{0.311} \\
\hline Low $\leq 2.5$ & 25 & 23 & & 10 & \\
\hline \multicolumn{6}{|l|}{ Preoperative PNI } \\
\hline High $>44.7$ & 28 & 26 & \multirow[t]{2}{*}{0.001} & 13 & \multirow[t]{2}{*}{0.003} \\
\hline Low $\leq 44.7$ & 15 & 9 & & 7 & \\
\hline \multicolumn{6}{|l|}{ Preoperative CEA } \\
\hline High $>5 \mathrm{ng} / \mathrm{mL}$ & 19 & 24 & \multirow[t]{2}{*}{0.89} & 9 & \multirow[t]{2}{*}{0.485} \\
\hline Low $\leq 5 \mathrm{ng} / \mathrm{mL}$ & 24 & 21 & & 10 & \\
\hline \multicolumn{6}{|l|}{ Preoperative CA 19-9 } \\
\hline High >37 U/mL & 31 & 15 & \multirow[t]{2}{*}{0.207} & 8 & \multirow[t]{2}{*}{0.13} \\
\hline Low $\leq 37 \mathrm{U} / \mathrm{mL}$ & 12 & 38 & & 23 & \\
\hline
\end{tabular}

CA 19-9, carbohydrate antigen 19-9; CAR, C-reactive protein/albumin ratio; CEA, carcinoembryonic antigen; NLR, neutrophil/lymphocyte ratio; PNI, prognostic nutritional index.

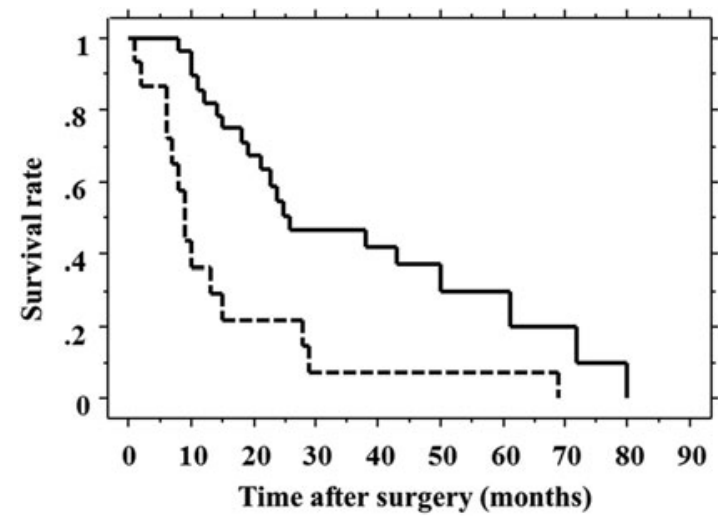

FIG. 1. Overall survival curves of patients. Twenty-eight patients with high PNI (solid line) had significantly better survival than 15 patients with low PNI (dotted line, $p=0.001$ ). PNI, prognostic nutritional index.
Also, some cases of POPF can be fatal. Soft pancreas or pancreatic thickness has been thought to be risk factors for occurrence of POPF. ${ }^{25,26}$ In our series, we found POPF in eight cases (18.6\%, PD: 5/28, 17.9\% and DP: $3 / 15,20 \%)$, however, we found no significant correlation between POPF and poor survival.

The preoperative estimation of risk factors for poor prognosis of patients with PDACs has been studied. Pathological tumor stage was reported as a strong prognostic factor for patients with PDACs, but preoperative serum tumor markers, such as CA 19-9 and pancreatic cancer-associated antigen (DUPAN-2), were not. ${ }^{4}$ However, clinicians may change the treatment strategy for patients with PDACs based on strong preoperative prognostic makers. For patients who have poor prognosis based on preoperative prognostic markers, we recommend chemoradiotherapy before radical surgery, or perhaps even avoiding the surgical procedure altogether because of the high incidence of postoperative complications. In this study, we retrospectively analyzed the prognostic markers of patients with PDACs 
Table 3. Multivariate Overall Survival Analysis of 43 Patients

\begin{tabular}{|c|c|c|c|}
\hline & $p$ & $\begin{array}{l}\text { Odds } \\
\text { ratio }\end{array}$ & $\begin{array}{c}95 \% \\
\text { Confidence } \\
\text { interval }\end{array}$ \\
\hline \multicolumn{4}{|l|}{ Operative procedures } \\
\hline $\begin{array}{l}\text { DP or total pancreatectomy vs. } \\
\text { SSPPD with or without portal } \\
\text { vein resection }\end{array}$ & 0.995 & 0.996 & $0.325-3.053$ \\
\hline \multicolumn{4}{|l|}{ Operation time } \\
\hline Long >297 min vs. short $\leq 297$ min & 0.062 & 2.625 & $0.954-7.194$ \\
\hline \multicolumn{4}{|l|}{ Tumor stages } \\
\hline Stage I and IIA vs. Stage IIB and III & 0.148 & 2.1 & $0.768-5.747$ \\
\hline \multicolumn{4}{|l|}{ Postoperative complication } \\
\hline Yes vs. no & 0.083 & 0.419 & $0.157-1.118$ \\
\hline \multicolumn{4}{|l|}{ Preoperative amylase level } \\
\hline High $>125 \mathrm{U} / \mathrm{L}$ vs. low $\leq 125 \mathrm{U} / \mathrm{L}$ & 0.4 & 0.617 & $0.201-1.899$ \\
\hline \multicolumn{4}{|l|}{ Preoperative CAR } \\
\hline High $>0.04$ vs. low $\leq 0.04$ & 0.025 & 2.895 & $1.142-7.339$ \\
\hline \multicolumn{4}{|l|}{ Preoperative NLR } \\
\hline High $>2.5$ vs. low $\leq 2.5$ & 0.103 & 0.373 & $0.114-1.22$ \\
\hline \multicolumn{4}{|l|}{ Preoperative PNI } \\
\hline High $>44.7$ vs. low $\leq 44.7$ & 0.003 & 6.803 & $1.919-24.39$ \\
\hline \multicolumn{4}{|l|}{ Preoperative CEA } \\
\hline High $>5 \mathrm{ng} / \mathrm{mL}$ vs. low $\leq 5 \mathrm{ng} / \mathrm{mL}$ & 0.829 & 0.914 & $0.403-2.072$ \\
\hline \multicolumn{4}{|l|}{ Preoperative CA $19-9$} \\
\hline High $>37 \mathrm{U} / \mathrm{mL}$ vs. low $\leq 37 \mathrm{U} / \mathrm{mL}$ & 0.746 & 1.231 & $0.35-4.335$ \\
\hline
\end{tabular}

at a single center. In doing so, we found that high CAR and low PNI were strong prognostic preoperative markers independently of tumor stage. Also, we found that postoperative complications did not influence the survival of the patients. Two markers of systemic inflammatory response, CRP and albumin, have been used in combination to diagnose not only chronic inflammation but also the nutritional status of cancer patients. In addition, pretreatment CAR was shown to be a significant prognostic indicator in various carcinomas. ${ }^{27-29} \mathrm{PNI}$ is based on albumin and absolute lymphocyte count, which are measured routinely in clinical practice. This index is designed to assess nutritional and immunological status, which may be useful in predicting prognosis. Moreover, a significant correlation between low PNI and poor survival has been reported in various cancers. ${ }^{16,30,31}$ In PDACs, Geng et al. ${ }^{31}$ reported that low PNI correlated significantly with shorter OS in patients with advanced pancreatic cancer. In this study, multivariate analysis identified PNI as an independent prognostic factor for OS. Thus, we recommended the preoperative assessment of CAR and PNI before planning the treatment strategies for patients with PDACs. Also, it may be possible that improvement of nutrition before surgery may improve patient's postoperative prognosis in PDACs.

\section{Conclusion}

High preoperative CAR and low PNI levels strongly correlated with poor survival in patients with PDACs. These markers should be assessed when selecting the treatment strategies for patients with PDACs.

\section{Acknowledgment}

We specially thank Dr. Hiroaki Saito (Department of Surgery, Division of Surgical Oncology, Faculty of Medicine, Tottori University, Yonago 683-8504, Japan) for analyzing the ROC curves.

\section{Author Disclosure Statement}

No competing financial interests exist.

\section{References}

1. Distler M, Pilarsky E, Kersting S, et al. Preoperative CEA and CA 19-9 are prognostic markers for survival after curative resection for ductal adenocarcinoma of the pancreas-a retrospective tumor marker prognostic study. Int J Surg. 2013;11:1067-1072.

2. Ben Q, An W, Wang L, et al. Validation of the pretreatment neutrophillymphocyte ratio as a predictor of overall survival in a cohort of patients with pancreatic ductal adenocarcinoma. Pancreas. 2015;44:471-477.

3. Marsoner K, Kornprat P, Sodeck G, et al. Pancreas cancer surgery in octogenarians-should we or should we not? Anticancer Res. 2016;36:1979-1984.

4. Yamamoto $\mathrm{T}$, Yagi $\mathrm{S}$, Kinoshita $\mathrm{H}$, et al. Long-term survival after resection of pancreatic cancer: a single-center retrospective analysis. World J Gastroenterol. 2015;21:262-268.

5. Sato D, Tsuchikawa T, Mitsuhashi T, et al. Stromal palladin expression is an independent prognostic factor in pancreatic ductal adenocarcinoma. PLoS One. 2016;11:e0152523.

6. Arnaud JP, Tuech JJ, Cervi C, et al. Pancreaticogastrostomy compared with pancreaticojejunostomy after pancreaticoduodenectomy. Eur J Surg. 1999;165:357-362.

7. Tabatabei SA, Hashemi SM. Pancreatic anastomosis leakage management following pancreaticoduodenectomy how could be manage the anastomosis leakage after pancreaticoduodenectomy? J Res Med Sci. 2015;20:161-164.

8. Pugalenthi A, Protic M, Gonen M, et al. Postoperative complications and overall survival after pancreaticoduodenectomy for pancreatic ductal adenocarcinoma. J Surg Oncol. 2016;113:188-193.

9. Ceppa EP, McCurdy RM, Becerra DC, et al. Does pancreatic stump closure method influence distal pancreatectomy outcomes? J Gastrointest Surg. 2015;19:1449-1456.

10. Akita $\mathrm{H}$, Takahashi $\mathrm{H}$, Gotoh $\mathrm{K}$, et al. Closure method for thick pancreas stump after distal pancreatectomy: soft coagulation and polyglycolic acid felt with fibrin glue. Langenbecks Arch Surg. 2015;400:843-848.

11. Yamashita S, Ishizawa T, Ichida A, et al. Advantages and disadvantages of prophylactic abdominal drainage in distal pancreatectomy. World J Surg. 2016;40:1226-1235.

12. Ubukata H, Motohashi G, Tabuchi T, et al. Evaluation of interferon- $\nu /$ interleukin-4 ratio and neutrophil/lymphocyte ratio as prognostic indicators in gastric cancer patients. J Surg Oncol. 2010;102:742-747.

13. Chua W, Charles KA, Baracos VE, et al. Neutrophil/lymphocyte ratio predicts chemotherapy outcomes in patients with advanced colorectal cancer. Br J Cancer 2011;104:1288-1295.

14. Ikeguchi M. Glasgow prognostic score and neutrophil-lymphocyte ratio are good prognostic indicators after radical neck dissection for advanced squamous cell carcinoma in the hypopharynx. Langenbeck's Arch Surg. 2016;401:861-866.

15. Nozoe $T$, Kohno M, Iguchi $T$, et al. The prognostic nutritional index can be a prognostic indicator in colorectal carcinoma. Surg Today. 2012;42:532-535.

16. Ikeguchi $M$, Ashida $K$, Saito $H$. New prognostic indicator is useful for predicting the survival of patients with unresectable advanced colorectal cancer. Hepatogastroenterology. 2015;62:61-64. 
17. Kanda M, Fujii $\mathrm{T}$, Takami $\mathrm{H}$, et al. Combination of the serum carbohydrate antigen 19-9 and carcinoembryonic antigen is a simple and accurate predictor of mortality in pancreatic cancer patients. Surg Today. 2014:44:1692-1701.

18. Wasif N, Ko CY, Farrell J, et al. Impact of tumor grade on prognosis in pancreatic cancer: should we include grade in AJCC staging? Ann Surg Oncol. 2010;17:2312-2320

19. Oida T, Kano H, Mimastu K, et al. Reconstruction following pancreaticoduodenectomy for patients who have previously undergone partial gastrectomy. Hepatogastroenterology. 2009;56:1199-1202.

20. Miki Y, Toyokawa T, Kubo N, et al. C-reactive protein indicates early stage of postoperative infectious complications in patients following minimally invasive esophagectomy. World J Surg. 2017;41:796-803.

21. Kawahara T, Yokomizo Y, Ito $Y$, et al. Pretreatment neutrophil-tolymphocyte ratio predicts the prognosis in patients with metastatic prostate cancer. BMC Cancer. 2016;16:111.

22. Chiaravalloti $A$, Fiorentini $A$, Palombo $E$, et al. Evaluation of recurrent disease in the re-staging of colorectal cancer by ${ }^{18} \mathrm{~F}$-FDG PET/CT: use of CEA and CA 19-9 in patient selection. Oncol Lett. 2016;12:4209-4213.

23. Kinaci E, Sevinc MM, Ozakay A, et al. Intraoperative acidosis is a new predictor for postoperative pancreatic fistula after pancreaticoduodenectomy. Hepatobiliary Pancreat Dis Int. 2016;15:302-309.

24. Fujino Y. Perioperative management of distal pancreatectomy. World J Gastroenterol. 2015;21:3166-3169.

25. Sugimoto $M$, Takahashi S, Kojima M, et al. In patients with a soft pancreas, a thick parenchyma, a small duct, and fatty infiltration are significant risks for pancreatic fistula after pancreaticoduodenectomy. J Gastrointest Surg. 2017. [Epub ahead of print]; DOI: 10.1007/s11605-017-3356-7.

26. Chang YR, Kang JS, Jang JY, et al. Prediction of pancreatic fistula after distal pancreatectomy based on cross-sectional images. World J Surg. 2017. [Epub ahead of print]; DOI: 10.1007/s00268-017-3872-3.

27. Gockel I, Dirksen K, Messow CM, et al. Significance of preoperative C-reactive protein as a parameter of the perioperative course and longterm prognosis in squamous cell carcinoma and adenocarcinoma of the oesophagus. World J Gastroenterol. 2006;12:3746-3750.

28. Hwang JE, Kim HN, Kim DE, et al. Prognostic significance of a systemic inflammatory response in patients receiving first-line palliative chemotherapy for recurred or metastatic gastric cancer. BMC Cancer. 2011;11:489.
29. Chen Z, Shao Y, Fan M, et al. Prognostic significance of preoperative Creactive protein: albumin ratio in patients with clear cell renal cell carcinoma. Int J Clin Exp Pathol. 2015;8:14893-14900.

30. Miyazaki T, Sakai M, Sohda M, et al. Prognostic significance of inflammatory and nutritional parameters in patients with esophageal cancer. Anticancer Res. 2016:36:6557-6562.

31. Geng Y, Qi Q, Sun M, et al. Prognostic nutritional index predicts survival and correlates with systemic inflammatory response in advanced pancreatic cancer. Eur J Surg Oncol. 2015;41:1508-1514.

Cite this article as: Ikeguchi M, Hanaki T, Endo K, Suzuki K, Nakamura S, Sawata T, Shimizu T (2017) C-reactive protein/albumin ratio and prognostic nutritional index are strong prognostic indicators of survival in resected pancreatic ductal adenocarcinoma, Journal of Pancreatic Cancer 3:1, 31-36, DOI: 10.1089/pancan.2017.0006.

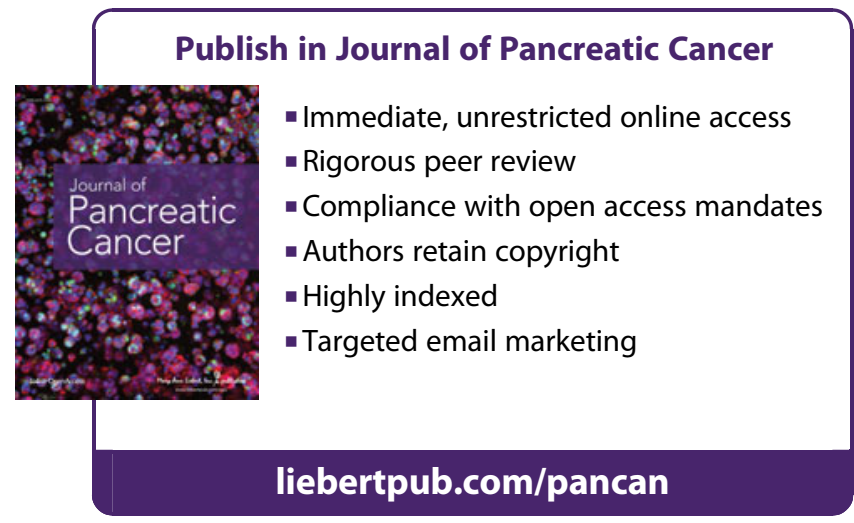

